\title{
Quality in pathology: how good is good? An introduction
}

\author{
Fred T. Bosman ${ }^{1} \cdot$ Hans-Anton Lehr ${ }^{2}$
}

Published online: 11 January 2016

(C) Springer-Verlag Berlin Heidelberg 2016

In any profession, medical or not, assurance of professional standards and of the quality of the end-product or the service rendered has become an essential requirement. This holds true also for the practice of pathology. It is the intention to cover in this issue of Virchows Archiv a variety of relevant aspects of quality assurance, directly pertaining to the practice of pathology, and also subjects indirectly related to pathology practice. This includes not only quality assurance in the laboratories (including issues in basic histology, immunohistochemistry, and molecular pathology) but also the question on how the quality of diagnostic practice at the microscope can be monitored, quality assurance in under- and postgraduate education and continuous professional development in pathology, sense and non-sense in laboratory accreditation, communication in pathology including the role of structured standard reporting, the role of the autopsy in quality of care assurance, quality assurance in postgraduate education, and last but not least quality assurance in pathology research. Aims and scope of this journal pretend to emphasize "evidence based" approaches. The subjects discussed in this issue, however, to a large extent express "what we think" or "what we are convinced of" as factual supporting evidence is often lacking, for want of studies or because of the nature of the subject. The

Fred T. Bosman

fred.bosman@citycable.ch

Hans-Anton Lehr

hansanton.lehr@yahoo.de

1 Institute of Pathology, University Medical Center (CHUV) of Lausanne, Lausanne, Switzerland

2 Institut für Pathologie im Medizin Campus Bodensee, Friedrichshafen, Germany papers therefore are mostly position papers, intended to stimulate reflection and discussion rather than providing practical solutions.

In the first paper in the series (DOI 10.1007/s00428-0151838-0) Clark describes how Lean management, an approach developed by the Toyota automobile company which creates value for the end-user by continuously improving operational effectiveness and removing waste, can be adopted in histopathology. It has been applied in departments throughout the world to simultaneously improve quality (reducing errors and shortening turnaround times) and lower costs (by increasing efficiency). The paper describes the key concepts and how these were adapted to histopathology, using a case study of Lean implementation and evidence from the literature. It discusses the benefits, limitations, and pitfalls encountered when implementing Lean management systems. The paper notably emphasizes the need for a change of mindset in the process of Lean implementation. The process goes way beyond introduction of a particular management system; it requires sustained specific people management skills in a working environment emphasizing continuous improvement. It requires significant efforts and means but its potential benefits are far reaching.

One of the established core techniques in diagnostic pathology is immunohistochemistry. In spite of the more than 30 years of its steady growth into automated immunostaining as we know it today, it remains a method beset with pitfalls, in terms of the quality of the reagents and of the analyte, of the protocols used and of the final interpretation of the obtained staining result. This has become even more important in the era of targeted therapy. Vyberg and Nielsen. (DOI 10.1007/s00428-015-1829-1) report on the experience gained with Nordic Immunohistochemical Quality Control (NordiQC), an international academic proficiency testing primarily aimed at assessing quality of the analytical phases of immunohistochemistry. A substantial proportion of the analyses performed (between 20 and $30 \%$ ) 
remain substandard due to problems with reagents (antibody specificity), analyte issues in terms of poor fixation, inadequate tissue processing or ineffective antigen retrieval, lack of sensitivity of the applied detection system, or non-specific staining. Most problems result in too weak or too strong staining, while in a minority of cases, non-specific staining is the problem. Individually tailored recommendations for protocol optimization and identification of best tissue controls constitute a core element in the quality assurance approach. Strikingly, over time (NordiQ has been in operation for over 12 years), the proportion of insufficient results has not decreased dramatically. This indicates that continuous proficiency testing is necessary and contributes significantly to the quality of the care provided.

Along the same vein, Tembuyser and Dequeker (DOI $10.1007 / \mathrm{s} 00428-015-1839-\mathrm{z}$ ) addresses quality assurance in molecular diagnostics. With the introduction of precision medicine, treatments targeting specific intracellular signaling pathways gone awry, optimal quality of the tests supporting treatment decisions has become essential: patients might not receive potentially effective treatment or, conversely, might be exposed to inefficient treatment accompanied by side effects at high cost for society. As in immunohistochemistry, the quality of the analyte (starting with pre-analytical factors such as sample collection) and the analyses performed by the laboratory but also interpretation in a clinical context and reporting of test results to clinicians are key elements to take into account. External effective quality assessment schemes are therefore of paramount importance. Effective quality assurance in molecular diagnostics requires attention to details at each level in a multidisciplinary approach. This review aims to provide an overview of good quality assurance practices and discusses certain risks and recommendations to promote and improve quality assurance for both diagnostic laboratories and for external quality assessment providers. A call is made for universally applicable quality assurance approaches and accreditation criteria.

Long-Mira et al. (DOI 10.1007/s00428-015-1837-1) reflect on accreditation in pathology. The intention of an accreditation procedure is to document good professional practice as defined by internationally established standards. Its finality, a document stating that the laboratory is accredited, provides recognition of the quality of the analyses performed by a laboratory and the care provided to patients. For Europe, the usual standard is an ISO 15189 norm. A problem of this norm is that it was originally conceived for clinical chemistry laboratories. Practice in a pathology laboratory, however, differs significantly from that in a clinical chemistry laboratory in that the final result is not a laboratory value but an expert medical diagnosis, which has required the eyes and expert judgment of a well-trained pathologist. Quality assurance of laboratory procedures which can be standardized is relatively straightforward. Quality assurance of observing histological slides and interpreting the findings in a clinical context is a totally different matter. In currently applied accreditation approaches, strong emphasis is on laboratory procedures, which undoubtedly are essential but not always decisive. A good pathologist can make a solid diagnosis on a relatively poor slide while a sub-par performing pathologist might not get to the right diagnosis on an absolutely impeccable slide. Requirements for accreditation, according to the ISO 15189 norm, include an operational quality management system and continuous control of the methods used for diagnostic purposes which sounds reasonable. And yet, some of the requirements stipulated in the accreditation standards and norms are perceived as arduous and even illadapted to or even useless in daily pathology practice. Hoffman et al. openly discuss their perception of what is right and wrong with the current approaches towards accreditation.

In the paper by Ellis and Srigley (DOI 10.1007/ s00428-015-1834-4), structured synoptic reporting in pathology is reviewed. The authors reason that, while key quality parameters in diagnostic pathology with direct impact on quality of care include timeliness, accuracy in terms of the diagnosis made according to existing guidelines and clarity in communication, worldwide developments in eHealth, and management of big data generate new requirements. Population level studies can be facilitated by structured reporting of pathology data in standardized electronic format and when combined with multiple health data sources through eHealth and data linkage, such reports become central to population-level quality monitoring, benchmarking, interventions, and benefit analyses in public health management. To be reliable at a global level, a single agreed international and evidence-based standard is needed, to ensure interoperability and comparability. The authors describe how this is developed for cancer reporting through the International Collaboration on Cancer Reporting (ICCR). The evidence presented supports the essential role of structured pathology reporting in quality improvement for both clinical care and for population-level health management. In spite of the overwhelming arguments in favor of synoptic reporting, its introduction will meet with some resistance. In this context, an important issue is heterogeneity: the morphological spectrum of disease at tissue level is impressive and it can be perceived as frustrating to see potentially relevant information lost by being forced to press every lesion into a preformatted category which might not be a perfect fit (be it the primary diagnosis, subtype, or stage information). The fundamental problem is that classifications press the diversity and complexity of nature into a rigid mold, which suggests that we fully understand what we are looking at while in reality much of the morphological 
manifestations of disease are still ill understood. A classical example of this problem are the numbers pathologists use as cut-off for diagnostic decisions: how many intraepithelial lymphocytes for a diagnosis of celiac disease and how many cycling (Ki-67 expressing) cells to qualify for chemotherapy in breast cancer or to move from grade I to grade II in case of a neuroendocrine tumor. Some argue that replacing a narrative diagnosis, which requires a high level of reflection, by pull down menus may lead to loss of accuracy and vigilance.

Lehr and Bosman (DOI 10.1007/s00428-015-1848-y) reflect on the role of the pathologist as communicator. Communication in pathology is not limited to formal (structured, synoptic, or narrative) reporting. While an essential element of good medical practice, also in pathology, communication skills are not easy to define, teach, or assess. The paper is therefore more of a personal reflection of the authors rather than a set of guidelines. The authors explore issues such as what should be provided by requesting physicians on the pathology request form, how pathologists should communicate to get to meaningful internal (interpathologist) consultation, the interaction between surgeon and pathologists around frozen section diagnoses, reporting styles in terms of a final diagnosis, how to communicate critical and unexpected findings, who should (or might) receive pathology reports and modalities and content of communication in case of an error or a technical problem. Even though recommendations in this domain cannot be considered as validated or even potentially amenable to validation, the paper does provide a set of more formal descriptions of what might be expected of a pathologist in terms of communication at large.

Along with pathology becoming a broadly recognized medical specialty, it became customary to submit (almost) any cell or tissue specimen removed from a patient for histopathological examination. In this era of shortage of pathologists, increasing complexity of the daily activities of a diagnostic pathologist with the introduction of molecular diagnostics and considerations of cost of health care, the question as to whether or not any specimen taken out of a patient should be submitted to pathological examination has become quite relevant. Damjanov et al. (DOI 10.1007/s00428-015-1801-0) address this issue. They argue that most surgical specimens deserve to be submitted for pathologic examination, as this may yield valuable new information relevant for the future treatment of the patient. That having been said, the authors concede that a small number of specimen types, of which pathology would provide limited or no clinically valuable information, might be put on a list of specimens "exempt from submission." Alternatively, some might be labeled as "for gross examination only." National regulatory bodies provide general orienting guidelines on how to deal with various specimens. The authors argue that the final decision on which specimens might be eliminated and which ones merit partial or full pathologic examination is to be taken through consensus agreement between clinical and laboratory physicians, while respecting general guidelines. The issue does stir up at times emotional discussions. One can argue that appendices, gallbladders, and the likes can potentially serve as an important tool of quality control in surgery, provided that the pathology report is accurate in calling what is normal "normal." Such feedback might improve clinical diagnostic skills. It is not rare, however, that (sometimes for billing reasons) "chronic unspecific inflammation" is the minimal diagnosis made. The list provided in the paper is not exhaustive: tonsils, adenoids, and placentas are not routinely submitted to pathologic examination in many countries. Can this be regarded as adequate? Some reason that leaving the decision to the clinician is likely to result in a biased selection and potentially a drop in the quality of care. The decision might be deferred to the pathologist but here financial incentives to perform a full examination might come into play. Part of the reasoning often is that relative to the total cost of health care, the cost of pathology is almost negligible and that this justifies an "(almost) everything is to be submitted" approach.

Van den Tweel and Wittekind (DOI 10.1007/s00428-0151833-5) review the position of the autopsy in terms of its contribution to quality of care. This does not only concern care in a direct sense but also indirectly in terms of the quality of medical education, to which autopsies potentially contribute significantly. The authors argue that studies continue to emphasize the importance of this "final consultation," also in this era of sophisticated imaging techniques. Discrepancies between ante mortem and postmortem diagnoses are still a reality. An important question is why autopsies have been on the decline for several decades now. The answer is not simple because not only the perception of the population at large but also that of the responsible physicians, who rely on sophisticated diagnostic tools, and of pathologists, who mostly no longer regard the autopsy as a core activity, have to be considered. Part of the problem might be the way in-hospital care is organized. Specialization in combination with working hour constraints has resulted in a situation in which one physician who is on duty when a patient dies might not know the case nor the family and lacks motivation to request an autopsy. Autopsies are often performed by junior residents who do not necessarily dispose of the experience required to provide clinicians with the most relevant answers. Complaining about declining autopsy rates has become a tantrum of pathology leaders (the more experienced pathologists sit in the committees and write the reviews on the subject), and it is almost politically incorrect to utter other views. At any rate, the future of the autopsy will also depend on how pathologists address the issues at stake. 
Two papers in this issue are dedicated to the role of pathology in research. The first one by Röcken (DOI 10.1007/ s00428-015-1857-x) discusses the role pathology has in clinical research, more in particular in clinical trials. Hopes and dreams on precision medicine, largely driven by "omics" data and targeted drugs in the pipeline of pharmaceutical companies, have generated a large number of clinical trials based upon new paradigms which explore efficacy of novel treatment regimens. In parallel, in these trials, often simultaneous assessment of the capacity of tissue-based biomarkers to predict response of individual patients (companion diagnostics) is included. The authors argue that pathologists play a key role in such trials. They are responsible for cutting edge diagnostics, for tissue processing compatible with molecular analysis later on and for selection of samples to be submitted for molecular analysis. This has been the case for academic pathology but in view of the evolution in cancer medicine, it is not unlikely that most cancer cases will at some point of time require molecular testing, to which every practicing pathologist should be apt to contribute. Pathologists do not only play a passive, supportive role. Given the complexity of cancer biology, it is important that pathologists are implicated in the initial phases of trial development, to contribute knowledge and insight. Schmitt et al. take this a step further in discussing the role of pathology in maintaining high-quality biospecimen repositories (DOI 10.1007/s00428-015-1825-5). High-quality human tissue samples linked to histopathological and clinical data are essential for basic pathogenetically oriented research as well as translational studies focusing on drug and biomarker development. This requires quality-assured tissue biobanks that provide such biospecimens to research, thereby increasing the impact and reliability of scientific results. Quality concerns do not only address the biomaterial specimen itself but also include all biobanking-related procedures. Expertise, infrastructure, documentation, and project management are keywords. The most demanding role for pathologists here is expert evaluation ("entry" and "exit" controls) of tissue specimens to guarantee highquality biomaterials.

In this issue, we have included one original paper, as it sheds light on an important issue: manpower planning in pathology and the effect of precision medicine on the pathology work force. Warth et al. (DOI 10.1007/s00428-015-1869-6) argue that novel guidelines, more detailed quality assurance, as well as intensified conventional, immunohistochemical, and molecular characterization of disease has an important impact on workload. Analysis of (pathologist) demographics and the evolution of diagnostic practice in an academic department in Germany, the latter providing evidence of a substantial increase in the number of slides per case, immunohistochemistry procedures and molecular analyses, suggests that we are facing an increasing gap between required and available work-force. It is the responsibility of pathology leadership to face this challenge and to find answers in supporting health-care policy makers to foresee effective measures.

The final two papers address quality assurance in postgraduate pathology education. Two authors discuss the situation in two countries in the European Union, of which one might have expected a sustained effort towards harmonization of postgraduate training. In reality, the ultimate responsibility for the training programs and for certification of medical specialists has remained at the national level and free movement of medical personnel has not led to uniform standards of training and certification. Van der Valk discusses the situation in The Netherlands (DOI 10.1007/s00428-015-1895-4), which is characterized by a combination of frequent "in service" evaluation at different levels (but without a final summative test) and strict criteria for accreditation of training departments which includes relatively frequent and in-depth site visits. Bailey (DOI 10.1007/s00428-015-1847-z) discusses the changes made to the curricula and assessment systems in the UK over the last decades, which have resulted in pathology specialty training programs which assess trainees on a regular (weekly or monthly) basis and in which multisource feedback is included, similar to what has been introduced in The Netherlands. An integral part of the UK system are summative tests at the end of first year, to test aptitude for the specialty, and after at least 2 or 3 years of training (the FRCPath examinations). In both countries, adequate coverage of curriculum content is monitored in workplace-based assessments and objective-structured pathology examinations and generic non-clinical attributes and behavioral skills such as leadership and management skills are included. A universal problem with these continuously increasing regulatory requirements engendering a multitude of assessment approaches is that, while additional information is generated, trainees might not acquire enough practical experience in the laboratory. One cannot do without regulations, but the balance between didactic teaching with formal assessment, and apprenticeship-style, practical hands-on learning is an issue that needs to be urgently addressed. 